Service social

\title{
Diversité et créativité en recherche qualitative
}

\section{Michel Dorais}

Volume 42, numéro 2, 1993

Recherche et pensée critique

URI : https://id.erudit.org/iderudit/706615ar

DOI : https://doi.org/10.7202/706615ar

Aller au sommaire du numéro

\section{Éditeur(s)}

École de service social de l'Université Laval

\section{ISSN}

1708-1734 (numérique)

Découvrir la revue

\section{Citer cet article}

Dorais, M. (1993). Diversité et créativité en recherche qualitative. Service social, 42(2), 7-27. https://doi.org/10.7202/706615ar

\section{Résumé de l'article}

Dans cet article, l'auteur dresse un panorama des différents types de recherche qualitative selon leur objet d'étude, leur mode de collecte des données, leur traitement de ces données et leur finalité. Il insiste plus particulièrement sur la construction empirique de la théorie, méthode d'analyse qui permet le développement de concepts et de théories à partir de la comparaison de cas étudiés, qu'ils soient similaires ou atypiques, Enfin, l'auteur aborde la question des différents critères de qualité de recherche, question primordiale pour une reconnaissance accrue du bien-fondé et du caractère scientifique de ces recherches. 


\section{$A R T|C| \& S$}

\section{Diversité et créativité en recherche qualitative}

Michel DORAIS

Travailleur social de formation Termine des études postdoctorales Université Lumière, Lyon

\section{INTRODUCTION}

[...] Il est dommage que nous n'ayons pas davantage d'ouvrages qui, sur la base d'une observation attentive et prolongée, décrivent avec sensibilité les aspects sociaux de la réalité qui échappent aux questionnaires et aux statistiques. Cette rareté s'explique par la vaste créance accordée au dogme selon lequel, d'une part, rien ne vaut la peine d'être connu qui ne peut être quantifié et, d'autre part, toute information qui s'insère dans un tableau devient par là même scientifique. [...] Afin d'éviter les malentendus, je dois une fois encore répéter que j'apprécie pleinement l'utilité de la quantification lorsque celle-ci se fait de façon sensée et honnête. Ce à l'égard de quoi je m'élève, c'est d'une part contre le tabou mortel qui veut que l'on ne s'intéresse qu'à ce qui peut être quantifié et, d'autre part, contre le respect superstitieux accordé à tous les griffonnages qui ressemblent à des mathématiques (Andreski, 1975: 122 et 126).

Tableaux, graphiques, coefficients de corrélation, etc., ne rendent pas par eux-mêmes une étude «scientifique». En fait, je dirais que toute étude du comportement humain qui néglige - et ne met pas en 
valeur - le côté subjectif des expériences est fondamentalement non scientifique (Ehrenreich, dans Hite, 1983: 17).

Ces deux citations nous plongent au cœur d'un débat qui n'a pas fini de sévir en sciences humaines: qualitatif versus quantitatif. Atteints d'un complexe d'infériorité face aux sciences supposées «pures», nombreux sont les chercheurs qui ont cru que le salut des sciences humaines passait uniquement par la recherche quantitative, au mépris de ce qui ne comportait ni statistiques ni mesures précises. À ceux et celles qui ont échappé à ce complexe, je veux ici montrer la richesse des possibilités offertes par la recherche qualitative. Pour ce faire, j'ai mis à contribution mon expérience de chercheur et de formateur en recherche qualitative. Quoi de plus normal, en pénétrant dans un domaine où la subjectivité occupe - enfin - la place qui lui revient, que de commencer par reconnaître la sienne?

\section{UNE PLURALITÉ DE POINTS DE VUE}

On oublie souvent qu'au début de ce siècle la recherche en sciences sociales était presque synonyme de recherche qualitative. Ce qui caractérise, dès lors, ce type de recherche, c'est l'approche des phénomènes humains ou sociaux par l'expérience sensible, en particulier celle des acteurs sociaux en cause. Berceau de la sociologie américaine, l'école de Chicago, de l'université du même nom, allait développer cette perspective et exercer une influence déterminante durant plusieurs décennies. Jusqu'à ce que, vers la fin des années trente, un fort courant quantitatif dispute au qualitatif son statut scientifique. Le déclin du qualitatif qui allait s'ensuivre dura environ trente ans. Reprenant petit à petit la légitimité qu'elle avait avant l'imposition du dogme quantitatif (auquel le professeur Andreski faisait précédemment allusion), la recherche qualitative a pris au fil des dernières années un nouvelle vigueur et adopté une pluralité de visages. Esquissons un bref panorama de la diversité issue de cette renaissance.

En simplifiant les choses, cinq aspects de la recherche qualitative pourraient aujourd'hui servir à en répertorier les différentes pratiques. Après avoir présenté brièvement chacun de ces aspects, nous les étayerons et les illustrerons tour à tour au fil du présent texte.

Disons tout d'abord que le type d'approche utilisée dans la recherche représente une première source de différenciation: s'agit-il, en effet, d'une recherche empirique, d'une recherche évaluative, $d^{\prime}$ 'une recherche-action ou d'une recherche introspective? Deuxièmement, le champ d'étude choisi peut couvrir un espace allant du très vaste au très restreint. Les objets d'étude en recherche qualitative ont traditionnellement été les cultures, les souscultures, les groupes, la comparaison entre plusieurs cas d'espèce, un seul cas considéré comme prototypique, un programme de services, une situation 
problème, voire l'introspection du chercheur lui-même. Les techniques de collecte de données utilisées constituent un troisième élément différenciateur: les principales techniques sont l'entretien non directif, I'observation participante, le questionnaire ouvert et la recherche documentaire. Une fois les données recueillies, leur mode de traitement dépend en grande partie des finalités visées par la recherche. Ce sont les quatrième et cinquième caractéristiques d'une recherche. Ainsi une recherche exploratoire ou descriptive utilisera, par exemple, la description phénoménologique ou l'histoire de vie: il s'agit avant tout de montrer un phénomène de l'intérieur. La recherche visant la classification, la comparaison ou l'interprétation compréhensive sera volontiers axée sur des analyses de contenu, de discours, d'attitudes, de conduites ou d'interactions: elle visera à comprendre une réalité par delà le sens commun qui lui est généralement attribué. Enfin, l'approche dite de construction empirique de la théorie permettra d'en arriver à une explication, à une modélisation et, ultimement, à une théorie empiriquement ancrée concernant l'objet d'étude.

Le tableau suivant situe chacun des cinq éléments que nous venons de voir: type de recherche, champ ou objet d'étude, technique de collecte des données, traitement des données et finalité poursuivie.

Recherches qualitatives

\begin{tabular}{|c|c|c|c|c|}
\hline Tyре & Empirique & Recherche-action & Évaluative & Introspective \\
\hline Objet & $\begin{array}{l}\text { Culture } \\
\text { Sous-culture } \\
\text { Groupe } \\
\text { Plusieurs cas } \\
\text { Un seul cas }\end{array}$ & Situation problème & Programme & Soi-même \\
\hline $\begin{array}{l}\text { Collecte } \\
\text { des données }\end{array}$ & \multicolumn{4}{|c|}{$\begin{array}{l}\text { Entretien libre } \\
\text { Observation participante } \\
\text { Questionnaire ouvert (oral ou écrit) } \\
\text { Recherche documentaire }\end{array}$} \\
\hline $\begin{array}{l}\text { Traitement } \\
\text { des données }\end{array}$ & \multicolumn{4}{|c|}{$\begin{array}{l}\text { Description phénoménologique } \\
\text { Histoire(s) ou récit(s) de vie } \\
\text { Analyse de contenu (incluant analyse de discours) } \\
\text { Analyse d'attitudes, de conduites, d'interactions } \\
\text { Construction empirique de théorie }\end{array}$} \\
\hline Finalité & \multicolumn{2}{|c|}{$\begin{array}{l}\text { Exploratoire/descriptive } \\
\text { Interprétative }\end{array}$} & \multicolumn{2}{|c|}{$\begin{array}{l}\text { Classificatoire/comparative } \\
\text { Explicative/théorique }\end{array}$} \\
\hline
\end{tabular}

Avant de passer en revue ces éléments de façon plus détaillée, une remarque s'impose: un choix a été fait de mettre l'accent sur la recherche de type empirique, tout en consacrant plus de place encore à ses aspects les moins connus, parmi lesquels la construction empirique de la théorie. 
Cet accent vise à pallier le peu de documentation disponible en langue française sur certains aspects de la recherche qualitative, alors que d'autres, sur lesquels nous passerons plus rapidement, ont déjà fait l'objet de nombreux écrits.

\section{LES TYPES DE RECHERCHE QUALITATIVE}

La recherche empirique, sur laquelle nous allons plus particulièrement nous arrêter dans les pages qui suivent, postule que la connaissance de tout phénomène s'acquiert par la pratique, c'est-à-dire par l'expérience ou par l'observation de ce phénomène. La recherche-action incorpore intervention et recherche sur un problème donné. Le chercheur devient ainsi acteur social voulant orienter l'action en tenant compte des buts de sa recherche et, réciproquement, I'acteur social devient chercheur voulant mettre à profit sa recherche dans l'action. La recherche évaluative a pour but de fournir les informations nécessaires à l'énoncé d'un jugement sur l'efficacité de programmes de services. Lorsqu'elle est de type qualitatif, elle s'attache à recueillir des informations non quantifiables, telles que la satisfaction, les écarts entre objectifs visés et objectifs atteints, les effets désirés versus les effets non désirés, etc. Quant à la recherche introspective ou réflexive (Tesch, 1990: 3341), qui tient jusqu'à un certain point de la philosophie appliquée, elle vise à comprendre un phénomène à partir de l'intuition et de la réflexion du chercheur, généralement à partir de sa propre expérience ou de celle des autres, alors partagée de façon empathique.

\section{LES OBJETS OU CHAMPS DE RECHERCHE}

La culture représente le champ d'investigation par excellence de l'étude monographique. Largement utilisée en anthropologie, en ethnologie et en sociologie, l'étude monographique implique généralement une enquête terrain permettant l'observation participante et le recueil de témoignages susceptibles de reconstituer dans sa totalité un ou plusieurs phénomènes relatifs à une société donnée (Dufour, 1991: 18-9). Les travaux précurseurs de Bronislaw Malonowski, puis ceux de l'école de Chicago (Coulon, 1992) donnent une bonne idée de cette orientation, qui s'attache au point de vue subjectif des acteurs sociaux à propos de ce qu'ils vivent.

Cette même école de Chicago allait d'ailleurs développer un champ d'intérêt plus restreint et plus original encore: la sous-culture. Qu'il s'agisse de culture de pauvreté, de déviance ou de marginalité, les sociologues de l'université de Chicago et leurs émules ont donné ses lettres de noblesse à la 
révélation des rapports sociaux ou interpersonnels inhérents à une condition sociale déterminée: les immigrants, les marginaux, les délinquants, etc. Les études de Howard Becker (1985) ou de Erving Goffman (1968 et 1975), par exemple, illustrent bien cette tendance.

Le groupe, qu'il soit primaire (la famille) ou secondaire (l'école ou le travail), naturel ou constitué aux fins de la recherche, a, pour des raisons évidentes, surtout intéressé les psycho-sociologues et les chercheurs en éducation (Poisson, 1990) ou en gestion du travail (Aktouf, 1987). L'étude de la dynamique des groupes, de l'attribution des rôles et des statuts à l'intérieur de ces derniers a donné lieu à une abondante littérature. Ce qui caractérise l'apport qualitatif à ce mouvement, c'est l'accent mis sur la description et la compréhension du vécu de ces groupes et de leurs membres.

La comparaison de cas constitue un autre domaine d'élection pour la recherche qualitative. Ces «cas » peuvent tout aussi bien être des sociétés, des organisations (l'étude de cas organisationnels ou institutionnels s'est avérée très populaire au cours des dernières années) que des individus. En comparant les données accumulées sur différents cas, l'étude comparative entend dépasser l'anecdotique et mettre à jour les régularités, les constantes et les dissemblances qui émergent d'eux (Bruyne, 1974: 215). Découvrir des convergences entre plusieurs cas singuliers (Lessard-Hébert, 1990: 165) s'avère généralement la raison d'être de leur addition et de leur comparaison. Nous reviendrons d'ailleurs plus loin sur cet élément très souvent présent, sinon déterminant, en recherche qualitative.

L'étude d'un cas unique s'apparente à l'étude monographique décrite ci-dessus, sauf qu'elle concerne une réalité plus restreinte: une organisation, une famille, un individu. Qu'elle soit comparative ou singulière, l'étude de cas se retrouve dans des domaines aussi divers que la psychologie clinique - on pense immédiatement à Freud -, la sociologie, l'administration, la pédagogie, la science politique, l'urbanisme, l'histoire et l'économie (Yin, 1984). Photographie prise sur le vif d'un phénomène, l'étude d'un cas unique vise généralement à jeter un éclairage nouveau sur un acte, une décision ou un ensemble de décisions et ce qui en est résulté (Yin, 1984: 22-23), que ce soit à échelle individuelle ou collective. II peut aussi arriver qu'un cas, estimé prototypique pour différentes raisons (par exemple, à la suite d'une recherche comparative préalable), soit scruté et remis en question dans le détail (par exemple, Stoller, 1984).

L'étude d'une situation problème est bien connue des chercheurs systémiques, interactionnistes ou stratégiques, pour qui ce qui importe c'est l'interaction ou la communication entre les parties intéressées par un problème donné. Pour peu que nous utilisions le terme dans son sens le plus large, ce type d'étude peut être apparenté à l'étude de cas, dont nous venons de parler. 
L'évaluation de programme fait souvent appel à la fois à des données mixtes, c'est-à-dire à la fois de type quantitatif et qualitatif (Patton, 1987: 65-69). Le volet qualitatif permet alors de tenir compte de l'aspect humain et relativement subjectif de dimensions à évaluer, telles que l'impression et la satisfaction des clientèles, la qualité des services, la motivation du personnel, etc.

Enfin, la recherche de soi sur soi est généralement apparentée à une recherche de nature philosophique ou encore à de l'introspection thérapeutique. De plus, elle est généralement liée à une description phénoménologique, élément que nous aborderons plus loin de façon plus détaillée.

\section{LES TECHNIQUES DE COLLECTE DE DONNÉES}

Une abondante littérature existant déjà sur les techniques de collecte de données, nous serons très bref à ce sujet.

Puisqu'il est peu directif, l'entretien libre est souvent un moyen d'explorer une réalité ou un champ peu étudié. II offre l'avantage de permettre une franche interaction entre le chercheur et le(s) sujet(s) de son étude. Évidemment, ce genre d'entretien est susceptible de présenter certains biais : parmi ces derniers, le facteur émotif présent dans toute relation humaine $\mathrm{n}^{\prime}$ est pas le moindre. Toutefois, cette technique offre l'heureuse possibilité de s'immerger dans le monde et dans la pensée d'un interlocuteur afin de mieux saisir son point de vue. Au demeurant, cela présente une certaine similitude avec l'entrevue en travail social: ne vise-t-elle pas, en premier lieu, à comprendre le point de vue de l'autre?

L'observation participante est née des toutes premières études anthropologiques. De nos jours, elle a connu beaucoup de popularité en éducation, notamment, et pour cause: la classe constitue un milieu clos qui se prête bien à l'observation. Fidèles aux enseignements de l'école de Chicago, des chercheurs ont toutefois la hardiesse d'explorer des réalités plus intimes, voire clandestines, par ce moyen: partage des tâches entre partenaires, interactions dans les bars et dans le milieu de la prostitution, par exemple (Welzer-Lang et al., 1993 et 1994). Comme son nom l'indique, l'observation participante vise à décrire la réalité observée « de l'intérieur », c'est-à-dire en adoptant le point de vue de personnes vivant cette réalité. Tenue d'un « journal de bord » détaillé et utilisation de matériel audiovisuel figurent parmi les techniques utilisées qui permettent de saisir sur le vif les composantes des phénomènes en action et les conséquences qu'ils produisent. Principal reproche fait à cette approche: chaque observation est, pour des raisons évidentes, difficile à reproduire, d'où un possible problème de transférabilité.

On pourra s'étonner de voir l'utilisation d'un questionnaire figurer parmi des moyens de collecte de données qualitatives. Pourtant, un question- 
naire composé de questions ouvertes peut fort bien servir de guide dans un entretien plus ou moins directif. Ce questionnaire peut alors être utile pour fournir des pistes repères aux chercheurs et aux répondants, de façon à faciliter la collecte et la comparaison des données sur des aspects précis de la réalité que I'on souhaite étudier. Évidemment, I'utilisation d'un guide d'entrevue en recherche qualitative requiert un certain doigté: il ne s'agit pas d'orienter des réponses, mais de les faciliter ou de les circonscrire autour de certains thèmes. Ayant utilisé cette méthode au cours d'une récente recherche (Dorais, 1991a, 1991b), I'auteur de ces lignes a pu mesurer la difficulté, mais aussi l'intérêt d'une telle entreprise.

Popularisée par les historiens et par certains sociologues, la recherche documentaire permet $\mathrm{d}^{\prime}$ amasser des documents aptes à restituer la teneur et la dynamique d'une réalité. Les travaux fondateurs de l'école de Chicago, menés par Thomas et Znaniecki sur l'émigration polonaise, notamment à l'aide de lettres échangées entre ces immigrants et leurs familles, ont fait époque. Une recherche documentaire qui réussit à «faire parler» les textes ouvre des perspectives tout aussi vivantes et passionnantes que d'autres types d'études. Outre les documents personnels, les rapports professionnels ou institutionnels et les articles de revues ou de journaux peuvent servir à photographier, en quelque sorte, un phénomène déterminé et les réactions qu'il suscite.

\section{LES MÉTHODES DE TRAITEMENT DES DONNÉES}

Indéniablement, c'est dans le traitement des données que le chercheur manifeste le plus volontiers sa créativité et son originalité. Cinq tendances principales se retrouvent dans la recherche actuelle. Notons qu'elles ne s'excluent pas forcément les unes les autres et peuvent, au contraire, être vues comme faisant partie d'une continuité.

La description phénoménologique soumet les données à deux procédures: une analyse inductive, au cours de laquelle «les thèmes, les catégories ou structures [...] doivent découler ou émerger des données» (Joshi, 1986: 49) et une «élucidation eidétique des phénomènes étudiés, c'est-à-dire la délimitation de leurs structures de base et de leurs constituants et référents essentiels, en vue d'établir des généralisations théoriques universelles» (Joshi, 1986: 50). En outre, I'approche phénoménologique «privilégie tout spécialement la perception que les sujets ont de la réalité étudiée. $C^{\prime}$ est fondamentalement ce qui distingue cette approche des toutes les autres en recherche qualitative» (Poisson, 1990: 27). Cela amène le chercheur à s'effacer le plus possible, y compris dans son questionnement et ses connaissances préalables, afin de laisser tout le champ libre aux sujets de la recherche et à leurs propres perceptions et analyses (à moins, bien sûr qu'il ne 
s'agisse d'une recherche sur soi). Aussi la méthode phénoménologique se veut-elle surtout descriptive, quoiqu'elle puisse mener à des insights et à des hypothèses très heuristiques, et jusqu'à un certain point généralisables.

Le récit ou l'histoire de vie est une technique de recherche par laquelle le chercheur vise à comprendre un milieu social ou des phénomènes sociaux essentiellement à partir de l'expérience de vie d'une personne, d'un groupe de personnes ou d'une communauté (Deslauriers: 1991). Selon I'un de ses spécialistes reconnus (Bertaux, dans Desmarais, 1986: 25), les récits ou histoires de vie sont susceptibles de remplir diverses fonctions: une première, descriptive ou exploratoire, permet de s'initier à une réalité et d'en découvrir les «lignes de force», c'est-à-dire les dynamiques; une seconde, analytique, sert à étayer, à compléter, voire à produire une théorie en passant des perceptions aux hypothèses; une troisième se situe sur le plan de la synthèse et rejoint dès lors le discours d'ordre théorique: on est alors placé devant un récit restitué de telle façon qu'il «parle par lui-même ». Deux écoles de pensée se dessinent lorsqu'il s'agit de traiter les données amassées par récit(s) ou histoire(s) de vie: ou bien le chercheur procède à une analyse de contenu plus ou moins classique (voir cet item ci-après), ou bien il s'attarde à la singularité de ce(s) récit(s) et à ce qu'elle révèle (Lewis, 1972; Catani, 1982). Bien qu'un seul récit de vie puisse, s'il est suffisamment éclairant, suffire à saisir une réalité, c'est, idéalement, le croisement de plusieurs récits qui, en dégageant certaines constantes, permettra d'exprimer le plus fidèlement possible, voire d'expliquer, la réalité des personnes interrogées. On parlera d'analyse verticale quand celle-ci concerne un récit particulier et d'analyse horizontale quand les récits sont comparés les uns aux autres. C'est précisément cette analyse horizontale qui permet à la méthode des récits de vie de prétendre à la scientificité:

Quand l'approche biographique porte sur plusieurs sujets très divers bien qu'appartenant à un même groupe et que l'on atteint une certaine récurrence des thèmes dans les entretiens, n'est-on pas en droit d'avancer que nous sommes alors en présence d'un échantillon représentatif ou, plus exactement, que cette récurrence est un indicateur de représentativité spécifique à ce type d'approche? C'est bien la position, nous semble-t-il, de Daniel Bertaux quand, après avoir souligné que la saturation ne s'obtient pas aussi facilement qu'on le pense, il écrit: « lorsque la saturation est atteinte, elle confère une base très solide à la généralisation ; à cet égard, elle remplit pour l'approche biographique très exactement la même fonction que la représentativité dans l'enquête par questionnaire (Le Gall, dans Deslauriers, 1987: 37-38).

L'utilisation d'histoires de vie offre, en outre, l'avantage de faire ressortir I'universel du singulier. Franco Ferrarotti (1983: 50-51) a bien saisi cet avantage lorsqu'il affirme: 
Une vie est une pratique qui s'approprie des rapports sociaux, les intériorise et les retransforme en structures psychologiques par son activité de destructuration-restructuration. [...] Combien faut-il de biographies pour atteindre une vérité sociologique, quel matériel biographique sera le plus représentatif et nous donnera le premier des vérités générales? Ces questions n'ont peut-être aucun sens. Parce que - en toute lucidité allons jusque-là - notre système social est tout entier dans tous nos actes, dans tous nos rêves, nos délires, œuvres, comportements, et I'histoire de ce système est tout entière dans I'histoire de notre vie individuelle. [...] Bien loin de refléter le social, I'individu se l'approprie, le médiatise, le filtre et le retraduit en le projetant dans une autre dimension, celle, en définitive, de sa subjectivité. Il ne peut en faire abstraction, mais il ne le subit pas passivement, au contraire, il le réinvente à chaque instant.

Comme l'a souligné Gaston Pineau, « le plus personnel est aussi le plus universel [...]. Ce paradoxe de I'universel dans le singulier pose selon nous la réalité épistémologique qui fonde la valeur scientifique des histoires de vie comme méthode de recherche. » L'auteur va jusqu'à conclure que «l'étude approfondie d'un seul cas particulier peut révéler plus de vérités universelles que la comptabilisation statistique de X cas particuliers » (Pineau, 1983: 124-125).

L'analyse de contenu (en y incluant I'analyse de discours) peut être d'ordre quantitatif ou qualitatif. Si sa version quantitative est la plus connue, sa version qualitative tente de mettre l'accent sur une «lecture seconde» (Pourtois, 1988: 199) du document écrit ou enregistré qu'il s'agit d'étudier. La répartition du contenu en catégories en est la première étape à suivre. D'après Aktouf (1987: 122), ces catégories doivent posséder les caractéristiques suivantes: exclusives, afin d'éviter les chevauchements, exhaustives, afin de couvrir la totalité du document, évidentes, afin d'assurer une certaine objectivité, et pertinentes par rapport aux objectifs de la recherche. La seconde étape consiste à retenir des indicateurs (sémantiques, linguistiques ou autres) qui permettront de dégager une seconde signification du contenu étudié, bref de détecter le «non-dit» compris dans le message. Le tout à travers une activité de codage au cours de laquelle finesse et perspicacité sont de rigueur. Une autre approche, celle-là beaucoup moins apparentée au quantitatif, exige du chercheur qu'il se mette à la place de l'informateur afin de capter ses intentions premières. Les travaux bien connus de Michel Foucault (1984) sur les discours autour de la sexualité dans la Rome et la Grèce antiques en fournissent un bon exemple. Foucault y tient compte non seulement du discours lui-même, mais du contexte socioculturel dans lequel il est produit, de la psychologie de l'auteur étudié, des influences littéraires ou sociales qu'il subit, etc.

L'analyse d'attitudes, de conduites ou d'interactions en recherche qualitative a surtout été mise de l'avant par l'école interactionniste symbolique 
(apparentée à l'école de Chicago), qui met l'accent sur le sens que les acteurs sociaux étudiés donnent aux situations sociales qu'ils rencontrent (Poisson, 1990: 26). L'interactionnisme symbolique postule que les conduites humaines sont observables à deux niveaux: celui du comportement et celui de sa signification. En somme, l'interaction sociale sert de révélateur au sens porté par le comportement (Denzin, dans Manis et Meltzer, 1978: 60), puisque les significations données aux choses et aux situations influencent les réactions face à ces choses et situations. Aussi, les études interactionnistes symboliques (et leurs très proches parentes, les études ethnométhodologiques) mettentelles l'accent sur les phases ou les processus présents par delà les habitudes et les comportements du quotidien. Les études de Howard Becker (1985) sur la déviance ou celles d'Erving Goffman sur les rites sociaux ou institutionnels en fournissent un bon exemple (Goffman, 1968, 1974, 1975). Comme le souligne Richard Lefrançois (dans Deslauriers, 1987: 148), leur approche

consiste en une observation attentive des interactions en émergence et des processus sociaux en présence. [...] Grâce notamment à l'observation participante, aux entretiens intensifs, aux récits de vie et aux autoconfessions, le chercheur tente de reconstituer ces réseaux de relations interpersonnelles dans le but de dégager le sens objectif de la situation sociale.

Dans leur ouvrage Qualitative Data Analysis, Matthew B. Miles et Michael Huberman (1984) s'attachent précisément à montrer comment la recherche qualitative peut arriver à produire des diagrammes, des matrices, des modélisations et des réseaux de causalités qui détaillent les interactions et expliquent les processus étudiés, rejoignant ainsi un souci de formalisme jusque-là davantage associé à la recherche quantitative.

La construction empirique de la théorie (CEDLT) est une méthode d'analyse qui a principalement été mise en forme par les sociologues américains Barney G. Glaser et Anselm L. Strauss (1967, 1978, 1990). Cette méthode consiste essentiellement à définir les éléments constitutifs et les interactions propres à une situation sociale donnée, à partir des données empiriques recueillies sur le terrain. Le but de la CEDLT étant de générer concepts, hypothèses puis théorie à partir d'études de terrain, sa logique sera, du moins dans un premier temps, de type inductif plutôt que logico-déductif. C'est en effet la comparaison constante et systématique des données et des cas recueillis qui permet de faire émerger les constantes et les divergences présentes dans le phénomène étudié, cela jusqu'à saturation, c'est-à-dire jusqu'à ce que toutes les données et catégories, anciennes et nouvelles, se recoupent. L'élaboration de catégories conceptuelles à partir des données, la formulation d'hypothèses sur la nature de ces catégories et sur les liens existant entre elles, et la vérification de ces hypothèses à partir de nouveaux cas (y compris des cas limites susceptibles de contredire la théorie émergente), voilà autant d'étapes caractéristiques de la CEDLT. Cette théorie sera jugée substantive si elle est circons- 
crite à un objet d'étude donné; formelle si elle peut s'appliquer à d'autres objets d'étude ou à un champ d'étude plus vaste (Glaser, 1978: 144).

Si on la compare à d'autres méthodes, la construction empirique de la théorie présente les caractéristiques suivantes (Dorais, 1991a, 1993):

1. Son but n'est pas de vérifier une ou plusieurs théories existantes, mais plutôt de générer de nouveaux concepts et de nouvelles hypothèses à partir de l'observation empirique, permettant ultimement l'émergence de nouvelles théories.

2. La logique d'analyse utilisée est, au départ, davantage de type inductif que de type logico-déductif. En effet, plutôt que d'établir a priori un cadre théorique rigide et des hypothèses à vérifier, le chercheur privilégie la démarche inverse ${ }^{1}$ : il observe d'abord la situation, ses acteurs et leurs interprétations. À partir de là, il tente d'établir des concepts, des hypothèses et des explications pertinentes, esquisses qu'il cherche ensuite à corroborer ou à réfuter tout au long de sa recherche.

3. La méthode utilisée pour arriver à ce résultat est de poser les données empiriques recueillies comme point de départ principal de la recherche, plutôt que de viser à confirmer ou à infirmer des hypothèses ou des théories préétablies. Cela ne signifie pas que les théories existantes soient ignorées; elles servent plutôt à clarifier, à comparer et à confronter la théorie émergente à mesure qu'elle se développe.

4. L'observation ou l'interview semi-dirigée sont les principaux moyens utilisés pour recueillir les données empiriques servant à la construction empirique de la théorie.

5. La comparaison constante et systématique (analyse transversale) entre les données recueillies chez des informateurs ou des répondants est la clé de voûte de cette méthode. Par exemple: y a-t-il des convergences entre les données? quelles sont les divergences? comment expliquer les unes et les autres? Contrairement aux autres types de recherche, la construction empirique de la théorie rend indissociables les processus de collecte des données, de leur codification ou annotation, puis de leur analyse comparative (qui consiste à comparer chaque source de données avec toutes les autres pour dégager certaines constantes - ou ruptures -, afin de confronter et de raffiner les hypothèses théoriques qui émergent à mesure qu'avance la recherche). Soulignons par ailleurs que la réduction des données par codification ou annotation s'effectue généralement de façon inductive, et non à partir de catégories prédéterminées.

1. Ce qui n'empêchera pas, au besoin, I'élaboration d'un cadre de référence ou cadre conceptuel théorique qui, évidemment, relèvera davantage du paradigme que de la théorie achevée. 
6. La théorisation est conçue comme un processus continu, alors que la recherche traditionnelle conçoit trop souvent la théorie comme un tout achevé, voire doctrinaire, dont l'élaboration est réservée à quelques «grands penseurs» (morts et enterrés, de préférence).

7. Ce qui rend scientifique la méthode de construction empirique de la théorie, c'est la recherche de saturation - c'est-à-dire de non-contradiction - des données interprétées par la théorie émergente (démarche qui rejoint quelque peu la logique de la loi des grands nombres utilisée en recherche quantitative).

8. Le chercheur travaille à partir d'un échantillon théorique ouvert, d'après certaines données topologiques initiales. Autrement dit, l'échantillon n'est pas arrêté définitivement à l'avance, puisque ce qui importe c'est moins le nombre de cas étudiés ou leur stricte représentativité que leur faculté de compléter ou de confronter les données déjà recueillies.

9. La théorie et la science sont dès lors conçues comme des champs ouverts à l'exploration et à la critique. Si le monde social qu'il s'agit de décrire est en mouvement continu, il n'y a pas de raison pour que nos explications de ce monde ne soient pas aussi en évolution, d'où la pertinence des théories empiriquement ancrées.

10. La méthode de construction empirique de la théorie est pluraliste, en ce sens qu'elle est appliquée de différentes façons par divers chercheurs, dans le cadre de recherches dissemblables.

Comme nous venons de le voir, ce qui contribue à rendre scientifique la construction empirique de la théorie, c'est la recherche de saturation des données jusqu'à leur redondance, grâce à la recherche active de « cas négatifs » qui remettent en question les représentations que se fait le chercheur en fonction des entretiens recueillis jusque-là. Cette méthode s'appelle l'induction analytique. Daniel Bertaux explique clairement ce en quoi consiste la recherche de saturation par induction lorsqu'il écrit:

Les répétitions ne peuvent manquer d'attirer l'attention [...]. C'est à partir d'elles qu'il faut développer la théorisation. Et si, une fois dégagé clairement le caractère de ce qui se retrouve dans de nombreux cas, il apparaît qu'il s'agit bien d'un "objet sociologique», $c^{\prime}$ est-à-dire qu'il s'agit de quelque chose qui relève du social et non du psychologique, du collectif et non de l'individuel, alors on peut affirmer avoir atteint un premier niveau de saturation. Une fois que la construction d'une représentation mentale a atteint, grâce à une première étape de saturation, une forme raisonnablement cohérente [...], il ne reste plus qu'à chercher systématiquement à la détruire: si elle résiste, elle aura prouvé sa solidité. [...] On cherchera donc systématiquement à rencontrer des « cas de figure » qui contrediraient le modèle. Il s'agit en général de personnes qui appartiennent à des 
catégories qu'on a mal ou peu explorées. [...] La recherche systématique de ce que Lindesmith a appelé les " cas négatifs», ceux qui contredisent le modèle provisoirement saturé, contribue à la fois à la vérification du modèle et à son raffinement. Menée systématiquement, [...], elle aboutit à la reconstruction du modèle (ou théorie) qui finit par atteindre sa forme stabilisée, définitive, celle de la véritable saturation. Et le miracle s'accomplit: [...] une description qui va en profondeur, au cœur des phénomènes, et qui n'aurait jamais pu être trouvée par le moyen de questionnaires. Ainsi est résolu le problème qui semblait insoluble et dont la non-résolution cantonnait toute approche qualitative au stade exploratoire: le problème de la généralisation sans échantillon représentatif (Bertaux dans Desmarais et Grell, 1986: 28-29).

L'induction analytique joue, en quelque sorte, le rôle « d'avocat du diable » dans tout processus de construction empirique de la théorie, complétant la démarche scientifique de cette dernière par un effort de réfutation des hypothèses envisagées jusque-là. Comme l'a si bien écrit Howard Becker, spécialiste reconnu en recherche qualitative:

Même si la biographie ne fournit pas, par elle-même, de preuve décisive en faveur d'une hypothèse, elle peut être un cas négatif qui nous oblige à déclarer inadéquate la théorie proposée. Dire cela, $c^{\prime}$ est avoir un point de vue sur la généralisation scientifique qui mérite quelques commentaires. Nous pouvons décider d'accepter une théorie si elle explique, disons, $95 \%$ des cas qui sont de son ressort. Beaucoup d'éminents savants le font. À l'inverse, on peut dire qu'une théorie qui n'explique pas tous les cas est inadéquate et que des facteurs autres que ceux proposés par la théorie sont à l'œuvre pour produire le résultat que nous voulons expliquer. C'est essentiellement une question de stratégie. Si nous admettons comme normale l'existence $d^{\prime}$ 'une exception à toutes les règles, nous ne chercherons peut-être pas d'autres facteurs explicatifs avec autant d'obstination que dans le cas inverse. Mais si nous considérons ces exceptions comme des réfutations potentielles de notre théorie, nous serons incités à les examiner de plus près. Plus fondamentalement, le cas négatif aidera une analyse scrupuleuse en suggérant la direction que doit prendre la recherche. L'examen du cas négatif révélera des propriétés différentes de celles de cas par ailleurs similaires, ou bien révélera des processus dont les aspects n'ont pas été entièrement compris (Becker, 1986).

Comment la construction empirique de la théorie et l'induction analytique se marient-elles concrètement? La méthode pourrait être décomposée en dix étapes (librement inspirées de Turner, 1981):

1. Élaboration de catégories conceptuelles à partir des données recueillies. Ce sont, en quelque sorte, les thèmes qui ressortent de l'analyse. 
2. Saturation des catégories conceptuelles, en accumulant pour chacune suffisamment de confirmations empiriques, jusqu'à ce qu'il soit clair que toutes les informations recueillies, anciennes et nouvelles, recoupent ces catégories (sinon, on les repense, on les remplace ou l'on en ajoute).

3. Définition de plus en plus précise des catégories conceptuelles, une fois qu'elles semblent relativement établies.

4. Utilisation de ces catégories comme guides dans la classification ou la reclassification du matériel recueilli, dans l'émergence de nouvelles catégories conceptuelles, et comme stimulant à la réflexion théorique.

5. Développement de liens entre les catégories conceptuelles et formulation d'hypothèses au sujet de la nature de ces liens. Cette étape figure parmi les plus décisives: quelles corrélations existe-t-il entre les divers éléments recueillis?

6. Vérification des hypothèses concernant les liens reliant les catégories conceptuelles, en tentant de spécifier les conditions dans lesquelles ces hypothèses et ces liens se vérifient.

7. Lorsque ces hypothèses ne conviennent pas à un ou plusieurs cas, les reformuler, les modifier ou tout simplement les abandonner au profit de formules plus heureuses. Procéder ainsi jusqu'à ce que les hypothèses du chercheur aient été testées avec un éventail suffisant de cas divers - y compris des cas apparemment atypiques - pour qu'une certaine généralisation soit possible.

8. Établissement de comparaisons avec d'autres hypothèses et théories existantes (qu'elles soient complémentaires ou concurrentes) : pourquoi la nouvelle théorie en train d'émerger serait-elle autant ou plus heuristique?

9. Vérification logique de la théorie émergente: tient-elle dans tous les cas, y compris dans des « cas limites » inclus ou exclus dans l'étude menée jusque-là? Cette recherche de confrontation de la théorie en émergence est primordiale: seul le succès de cette démarche pourra permettre de valider les résultats obtenus.

10. Unification et simplification de la théorie émergente, afin que tous ses éléments s'intègrent harmonieusement, sans contradiction.

\section{LES FIN ALITÉS}

Comme nous l'avons vu dans la section précédente, les recherches qualitatives peuvent viser tout autant à l'exploration, la description, la classification, la comparaison, l'interprétation ou l'explication du phénomène étudié. 
Un continuum peut d'ailleurs être imaginé avec, à un pôle, la finalité exploratoire ou descriptive, à l'autre pôle, la finalité explicative/théorique et, entre les deux, les finalités classificatoire/comparative et interprétative (qui, d'une part, prennent pied sur la description et, d'autre part, participent à l'explication du phénomène mais sans forcément prétendre à une intégration ou à une élaboration théorique formelle). On notera que ces finalités, très souvent, se recoupent et s'additionnent: comment, en effet, classifier, interpréter ou expliquer sans d'abord décrire?

La finalité exploratoire ou descriptive est sans aucun doute la plus courante en recherche qualitative. Bien décrire une réalité, c'est déjà contribuer à mieux la connaître et la faire comprendre. Cependant, leurs aspects purement descriptifs ont parfois jeté le discrédit sur certaines recherches qualitatives en remettant en question leur apport à l'avancement de la connaissance scientifique. II n'en demeure pas moins que la valeur heuristique d'un rapport de recherche exploratoire ou descriptive peut s'avérer déterminante lorsqu'il s'agit d'étudier un phénomène récent, peu connu ou changeant, comme le sont tant de phénomènes sociaux. De plus, une bonne description terrain, qui arrive à déterminer les composantes d'un phénomène, peut très bien servir de tremplin pour effectuer des recherches ultérieures aux visées classificatoires, comparatives, interprétatives ou explicatives.

Comme l'a fait remarquer Madeleine Grawitz (1990: 453), la classification représente un premier effort d'abstraction dans le traitement des données. Mettre en ordre et catégoriser permet en outre de comparer. La catégorie et le type sont les principales formes de classification. La catégorie classe, ordonne de façon plus ou moins abstraite, alors que le type, plus concret, définit. Autrement dit, comme l'écrit Grawitz (1990: 454), "la catégorie distingue pour rassembler à l'horizontale alors que le type sélectionne pour particulariser, le plus souvent dans un mouvement vertical ». La classification, souvent utilisée en sciences naturelles, peut s'avérer utile en sciences humaines lorsqu'il s'agit d'ordonner la connaissance à propos d'un objet ou d'un champ d'étude suffisamment décrit, mais insuffisamment compris dans ses liens (différences ou ressemblances) avec d'autres objets ou d'autres champs auxquels on peut le comparer.

La psychologue Mireille Lafortune explique bien la notion d'interprétation en recherche qualitative lorsqu'elle écrit:

[...] il s'agit à la fois de faire sortir le sens d'une chose de l'obscurité et aussi de comprendre la production d'un autre, en l'occurrence ici, la production d'un sujet qui répond aux questions d'un chercheur. [...] Maud Manoni rappelle qu'interpréter c'est aller au-delà ou en deçà du contenu manifeste; c'est repérer le sens d'une conduite, d'un dire, d'un phénomène (Lafortune, $1989: 145)$. 
Lorsqu'il travaille à une telle recherche, le chercheur se fait forcément interprète afin de faire parler les faits (ou les paroles, les mots) au-delà de l'apparence et du sens commun. Généralement, l'adhésion à une école de pensée théorique ou, à tout le moins, à un cadre de référence paradigmatique guide cette interprétation (la grille psychanalytique en psychologie constitue un exemple maintes fois rencontré).

La finalité explicative et théorique n'est pas si fréquemment atteinte en sciences sociales, que ce soit en recherche quantitative ou qualitative. En effet si, à l'instar de P. de Bruyne et autres (1974: 107), on définit la théorie comme un système intégré de propositions (prémisses, concepts, hypothèses et conséquences) ayant des relations logiques entre elles, on doit reconnaître que peu de résultats de recherche s'y conforment en tout point. Cela dit, les modélisations et les explications, même partielles, produites par une recherche contribuent souvent à augmenter le corpus théorique dans un champ donné, ce qui peut permettre d'élaborer des théories générales plus pertinentes, qu'elles soient formelles ou substantives.

\section{LES CRITÈRES DE QUALITÉ EN RECHERCHE Q UALITATIVE}

Tout comme la recherche quantitative, le qualitatif possède aussi ses standards de qualité. Jean-Marie Van der Maren (cité dans Pourtois, 1988: 119) émet quatre critères de validation en recherche qualitative: la crédibilité, la transférabilité, la constance ou cohérence interne et la fiabilité. Ces critères recoupent d'ailleurs ceux émis par Alex Muchielli dans un plus récent ouvrage (1991).

La quantité, la diversité, la qualité et surtout la confrontation critique des données amassées (confrontation avec d'autres chercheurs, avec d'autres écrits ou avec les répondants eux-mêmes) contribuent à augmenter la crédibilité de la méthode de collecte de données retenue. II en va de même pour I'analyse de ces données: par exemple, cette dernière recoupe-t-elle des hypothèses déjà disponibles ou en diverge-t-elle? Ces divergences sont-elles justifiées de façon convaincante? Comment réagissent les répondants à l'analyse faite de leur situation ou de leurs propos (introduisant ainsi une rétroaction critique sur notre analyse)? On parlera de validité phénoménologique ou d'acceptation interne lorsque les acteurs en cause dans le phénomène étudié corroborent l'analyse proposée par le chercheur; et de validité référentielle ou de confirmation externe, lorsque les résultats de la recherche présentent une certaine continuité ou une cohérence relative avec des recherches ou théories couvrant le même champ.

La transférabilité concerne la généralisation des résultats obtenus à partir d'autres contextes ou échantillons que ceux analysés. L'échantillon théorique, 
souventes fois utilisé en recherche qualitative, vise précisément à retenir des cas - qu'ils soient typiques ou atypiques - susceptibles de se révéler heuristiques et, additionnés les uns aux autres, jusqu'à un certain point représentatifs de la réalité étudiée. L'échantillon théorique est en effet considéré comme étant saturé lorsque l'ajout de nouveaux cas ne procure plus de nouvelles données significatives.

La constance ou cohérence interne tient dans la continuité perceptible entre les données amassées et l'analyse construite sur ces données. La méthode de triangulation, qui consiste à faire participer au moins deux chercheurs (de préférence indépendants les uns des autres) afin de s'assurer que des biais ne viennent pas déformer le passage des données à l'analyse, peut aider à la constance interne. Mais c'est surtout la description détaillée des méthodes et de la procédure employées pour récolter les données et les interpréter, voire pour rédiger le rapport de recherche, qui permet le mieux d'apprécier le cheminement suivi par le chercheur, sa manière d'opérer et l'objectivation qu'il fait de sa propre subjectivité.

Enfin, la fiabilité jauge l'indépendance des résultats par rapport aux croyances professées par le chercheur. Cela concerne la transparence et I'honnêteté du chercheur: énonce-t-il ses présupposés théoriques? cherchet-il, plus ou moins consciemment, à les conforter plutôt qu'à les confronter? La triangulation théorique, qui consiste à comparer et à discuter ses propres résultats à la lumière de ceux de chercheurs d'écoles de pensée différentes, constitue aussi une façon d'accroître la fiabilité.

Passons maintenant en revue les critères de qualité plus particuliers aux cinq méthodes d'analyse précédemment répertoriées, soit la description phénoménologique, les histoires ou récits de vie, I'analyse de contenu, I'analyse d'attitudes, de conduites ou d'interactions et la construction empirique de la théorie.

La principale critique faite à la description phénoménologique tient à son recours exclusif à la subjectivité des sujets étudiés. Or, comme le souligne Christian Lalive d'Épinay (1985), au lieu de voir ce dont nous prive la subjectivité, nous pouvons tout aussi bien voir ce qu'elle nous apporte: un matériel d'une grande richesse pour comprendre le point de vue de l'acteur social. Ultimement, c'est le choc des intersubjectivités - celle du chercheur et celle du lecteur de son rapport de recherche - qui jaugera la vraisemblance du tableau impressionniste peint par l'étude phénoménologique.

La difficulté de l'analyse des histoires ou récits de vie a trait au caractère forcément subjectif et incomplet de ces derniers. Subjectif parce que le répondant sélectionne nécessairement ce qui est digne d'être raconté et le chercheur ce qui mérite d'être retenu; incomplet parce que ce choix est immanquablement lié à des contingences d'espace et de temps - on ne peut tout raconter, ni tout se remémorer. Toutefois, cette sélectivité, loin d'être 
jugée négative, peut paradoxalement s'avérer en elle-même porteuse d'informations: pourquoi tel événement paraît-il plus significatif ou déterminant qu'un autre? Même en admettant que la mémoire soit sélective et interprétative dans sa reconstruction et sa retransmission du passé, la reconstitution de ce dernier fournit précisément des indications sur la signification et I'importance que l'individu accorde aux choses et aux événements. L'histoire de vie rapportée par la personne ne reflète-t-elle pas la somme des interactions qui l'ont influencée de façon significative? Comme l'a écrit le philosophe Hilary Putnam (1990), il n'existe de vérité qu'interprétative et de réalité que contextuelle. Paradoxalement, notre vision subjective de la réalité procure cependant de précieux éléments d'information objective sur qui nous sommes, quelles influences nous avons subies et comment nous percevons les autres. En somme, les limites de cette approche constituent aussi ses possibilités. L'apport de matériaux documentaires « objectifs », le croisement de récits (d'une même personne à des moments différents ou de personnes présentant un vécu similaire), la relecture critique et, éventuellement, I'enrichissement de son récit par le répondant, voilà autant de façons « d'objectiver» la restitution d'un récit.

L'utilisation de codification afin de parvenir à établir des catégories explicites exige du chercheur qui utilise l'analyse de contenu le recours à un jugement subjectif. II existe deux façons de vérifier la fidélité du chercheurcodeur: la fidélité intercodeur vérifie l'uniformité du jugement de plusieurs codeurs sur les mêmes données et la fidélité intracodeur teste l'uniformité du jugement d'un même codeur dans l'espace et le temps. Le jugement inhérent au codage implique souvent le recours à des concepts connus ou à des théories existantes qui, dans une certaine mesure, peuvent « forcer » les données à entrer dans des catégories prédéterminées: encore là, l'important pour le chercheur est d'identifier très clairement ses références théoriques ou paradigmatiques et d'accepter de les remettre en question à partir de ses données plutôt que de forcer ces dernières à s'y soumettre.

En ce qui concerne les recherches comportant des analyses d'attitudes, de conduites ou d'interactions, les critères de qualité émis par le sociologue interactionniste Norman K. Denzin (dans Manis, 1978) s'avèrent très pertinents. Ces critères sont les suivants:

- Les données et les observations scientiques doivent d'abord être colligées indépendamment de leur interprétation.

- Tout chercheur porte certaines pré-notions, que reflète sa méthode de recherche et dont il doit savoir reconnaître les limites.

- Les significations et les interactions doivent être combinées avant que l'investigation soit complétée.

- La démarche précédente permettra au chercheur de prendre constamment en compte la vision du monde des sujets qu'il étudie - ce qui ne 
signifie pas qu'il doive lui-même renoncer à maintenir une distinction entre sens commun et conception scientifique de la réalité.

- Les méthodes de recherche doivent être capables de refléter tout aussi bien le changement que le statu quo présent dans un phénomène.

- Le propre d'un concept est d'abord d'être heuristique; le propre d'une théorie est d'abord d'être limitée à un objet formel ; les causalités doivent être interactives et universalisables.

Enfin, les méthodologues Anselm Strauss et Juliet Corbin (1990) émettent les sept critères suivants afin de s'assurer que toute théorie empiriquement fondée soit suffisamment explicite pour répondre aux canons de rigueur scientifique:

1. Les concepts doivent être générés par la recherche. Idéalement, on devrait être en mesure, à la lecture du rapport de recherche, de voir comment ces concepts ont été produits à partir des données.

2. Les concepts doivent être systématiquement reliés les uns aux autres. Et, encore là, ces liens doivent être perceptibles à partir des données qui ont permis de les dégager.

3. Pour être satisfaisante, la théorie doit avoir un pouvoir explicatif suffisant.

4. Le chercheur doit spécifier son objet d'étude et les limites d'application de sa théorie.

5. À cet effet, le contexte dans lequel la recherche a été menée doit être explicité.

6. La construction empirique de la théorie consiste à expliquer des processus, non seulement selon des stades ou des phases, mais aussi en fonction de la fluidité et du mouvement.

7. Les résultats de la recherche doivent être signifiants, c'est-à-dire apporter quelque chose de nouveau à la connaissance d'un phénomène.

\section{CONCLUSION}

À la lueur des idées émises dans ce texte, on voit combien la recherche qualitative, en dépit de sa diversité, possède des modes de collecte et de traitement de données qui lui sont propres et qui tendent à se raffiner à mesure que se répand sa pratique. Elle possède aussi des critères de qualité qui rehaussent de plus en plus son niveau de scientificité à mesure qu'ils sont appliqués. Pour qui veut relever le double défi de la rigueur et de la créativité, la recherche qualitative offre un intérêt non négligeable. Elle permet en effet de faire le lien entre l'imagination, l'audace de la découverte et la démarche scientifique. 
À I'heure où les problèmes psychosociaux se complexifient et où leur solution exige des vues originales, cette approche ouvre grande la porte aux chercheurs en quête d'idées et d'explications nouvelles.

\section{Références bibliographiques}

ANDRESKI, S. (1975). Les Sciences sociales, sorcellerie des temps modernes? Paris: PUF.

AKTOUF, O. (1987). Méthodologie des sciences sociales et approche qualitative des organisations. Sillery: Presses de I'Université du Québec.

BECKER, H. (1985). Outsiders. Paris: A.M. Métaillé.

BECKER, H. S. (1986). "Biographie et mosaïque scientifique», Actes de la recherche en sciences sociales, vol. XX, $\mathrm{n}^{\circ}$ 62-63: 107.

BERTAUX, D. (1976). Histoires de vies ou récits de pratiques? Paris: CORDES.

CATANI, M. (1982). Tante Suzanne: une histoire de vie sociale. Paris: Méridiens.

COUlON, A. (1992). L'École de Chicago. Paris: PUF, coll. «Que sais-je?».

DE BRUYNE, P. et al. (1974). Dynamique de la recherche en sciences sociales. Paris: PUF.

DESLAURIERS, J.-P. (1987). Les méthodes de la recherche qualitative. Sillery: Presses de l'Université du Québec.

DESLAURIERS, J.-P. (1991). Recherche qualitative: guide pratique. Montréal : McGrawHill.

Desmarais, D. (1986). Les récits de vie. Montréal : Éd. Saint-Martin.

DORAIS, M. (1991a). Les parcours dynamiques de la sexualité masculine. Thèse de doctorat en service social. Sainte-Foy: Université Laval.

DORAIS, M. (1991b). Tous les hommes le font: parcours de la sexualité masculine. Montréal: Le Jour/VLB éditeur.

DORAIS, M. (1993). «Une expérience de recherche qualitative: la méthodologie de Tous les hommes le font», dans la Revue sexologique, vol. 1, $\mathrm{n}^{\circ} 1$, printemps 1993.

DuFOUR, S. (1991). L'enquête terrain en sciences sociales. Montréal : Éd. Saint-Martin.

FerRarotti, F. (1983). Histoire et histoires de vie. Paris: Méridiens.

FOUCAULT, M. (1984). Histoire de la sexualité. Paris: Gallimard, 3 tomes.

GAUTHIER, B. et al. (1986). Recherche sociale. Sillery: Presses de I'Université du Québec.

GlaSER, B. (1978). Theoretical Sensitivity. San Francisco: University of California.

GLASER, B. et A. STRAuSS (1967). The Discovery of Grounded Theory: Strategies For Qualitative Research. New York: Aldine de Gruyter.

GOFFMAN, E. (1968). Asiles. Paris : Minuit.

GOFFMAN, E. (1974). Les rites d'interaction. Paris: Minuit.

GOFFMAN, E. (1975). La mise en scène de la vie quotidienne. Paris: Minuit, 2 tomes.

GraWitZ, M. (1990). Méthodes en sciences sociales. Paris: Dalloz. 
HITE, S. (1983). Le Rapport Hite sur les hommes. Paris: Robert Laffont.

JOSHI, P. et A. BACHELOR (1986). La méthode phénoménologique de recherche en psychologie. Sainte-Foy: Presses de l'Université Laval.

LAFORTUNE, M. (1989). Le psychologue pétrifié. Montréal: Louise Courteau.

LALIVE D'ÉPINAY, C. (1985). "Récit de vie et projet de connaissance scientifique », Recherches sociologiques, vol. XVI, $\mathrm{n}^{\circ} 2$.

LAPERRIÈRE, A. (1982). "Pour une construction empirique de la théorie: la nouvelle école de Chicago », dans Sociologie et Sociétés, vol. XIV, $\mathrm{n}^{\circ} 1: 35$.

LESSARD-HÉBERT, M. (1990). Recherche qualitative: fondement et pratiques. Montréal : Agence d'Arc.

LEWIS, O. (1972). Les enfants de Sanchez. Paris : Gallimard.

MANIS, J.G. et B.N. MELTZER (1978). Symbolic Interaction. Boston: Allyn \& Bacon.

Miles, M.B. et A.M. HubERMAN (1984). Qualitative Data Analysis. Beverly Hills: Sage. MuCHIELLI, A., (1991). Les méthodes qualitatives. Paris: PUF, coll «Que sais-je?».

OuELLET, A. (1982). Processus de recherche: une approche systémique. Sillery: Presses de l'Université du Québec.

Ouellet, A. (1990). Guide du chercheur. Boucherville: Gaëtan Morin.

PATTON, M.Q. (1987). How to Use Qualitative Methods in Evaluation. Newbury Park: Sage.

PINEAU, G. (1983). Produire sa vie. Montréal : Éd. Saint-Martin.

POISSON, Y. (1990). La recherche qualitative en éducation. Sillery: Presses de I'Université du Québec.

POURTOIS, J.-P. et H. DESMET (1988). Épistémologie et instrumentation en sciences humaines. Liège: Pierre Mardaga.

Putnam, H. (1990). Représentation et réalité. Paris: Gallimard.

SELLTIZ, T. et al. (1977). Méthodes de recherche en sciences sociales. Montréal : HRW.

StOlLeR, R. (1984). L'excitation sexuelle. Paris: Payot.

StRAUSS, A. et J. CORBIN (1990). Basics of Qualitative Research. Newbury Park: Sage.

TAYLOR, S.J. et R. BODGAN (1984). "Selecting Informants», dans Introduction to Qualitative Research Methods. New York: John Wiley \& Sons, p. 83 et s.

TESCH, R. (1990). Qualitative Research: analysis types and software tools. Bristol: Falmer Press.

TURNER, B.A. (1981). «Some Practical Aspects of Qualitative Data Analysis: One Way of Organizing the Cognitive Processes Associated with the Generation of Grounded Theory«, dans Quality and Quantity, Amsterdam : Elsevier Scientific Publishing Co., $\mathrm{n}^{\circ} 15: 23$.

WELZER-LANG, D. et J.-P. FILIOD (1993). Les hommes à la conquête de l'espace... domestique: du propre et du rangé. Montréal : Le Jour/VLB éditeur.

Welzer-LanG, D., A. Barbosa et L. MATHieu (1994). Prostitution: les uns, les unes et les autres. Paris: A. M. Métaillé.

YIN, R. (1989). Case Study Research: Design and Methods. Newbury Park: Sage. 